\title{
Summary of Six Recent Studies Every Practicing Obstetrician Gynecologist Should Know
}

\author{
Geetha Balsarkar $^{1}$
}

Received: 25 October 2021 / Accepted: 25 October 2021 / Published online: 15 November 2021

(c) Federation of Obstetric \& Gynecological Societies of India 2021

Season's greetings to all FOGSI members.

I wish that in FOGSI family all are healthy, safe and enjoying Diwali festivities with family and friends. Although we are seeing a reduction in the number of COVID cases in India, it is very important that we be safe and follow hand sanitization and social distancing to protect ourselves.

Diwali reminds me of the number of years that as Resident Doctors we could not go home to enjoy the festival. Friends and colleagues then enjoy the festivities with togetherness, and new families are formed. Unity in diversity can be easily seen in any medical college hostel in the country.

We entered the field of Obstetrics and Gynecology very keen to learn, always do well for patients and become the practicing consultants we are today. We believe in reproductive rights of our women and evidence-based medicine: Together, these help us provide optimal care. Staying up to date with the literature is very important for any clinician who.

These are summary of six recent studies every practicing obstetrician gynecologist should know.

1. Gestational age at time of COVID-19 and outcome (September 2021)

In an international retrospective cohort study evaluating obstetric and neonatal outcomes of nearly 400 SARS-CoV2-positive patients according to their gestational age at the time of infection, infection after 20 weeks increased the risk for adverse obstetric outcomes and infection after 26 weeks

Geetha Balsarkar is a Professor, Department of Obstetrics and Gynecology, Seth G. S. Medical College, Nowrosjee Wadia Maternity Hospital, Mumbai, India.

Geetha Balsarkar

gdbalsarkar@yahoo.com

1 Department of Obstetrics and Gynecology, Seth G. S. Medical College, Nowrosjee Wadia Maternity Hospital, Mumbai, India increased the risk for adverse neonatal outcomes [1]. Given these findings, the increased severity of COVID-19 in pregnant women, the good safety profile of SARS-CoV-2 vaccines in pregnancy and the time it takes to become fully vaccinated, we recommend vaccination as soon as possible for women planning to conceive and pregnant women.

2. COVID-19 vaccination does not increase risk for miscarriage (September 2021)

Evidence of the safety of COVID-19 vaccination in pregnancy continues to accrue. In one study involving nearly 2500 pregnancies, the age-standardized cumulative risk of miscarriage was $12.8 \%$ individuals who received a mRNA COVID-19 vaccine preconception or prior to 20 weeks of gestation, which is similar to the expected miscarriage rate in the general obstetric population [2]. In another study including over 105,000 pregnancies, individuals who experienced miscarriage had similar odds of exposure to a COVID-19 vaccine in the prior 28 days as those with ongoing pregnancies [3]. Results were consistent with either mRNA-1273 or BNT162b2 vaccine exposure; risks specific to Ad26. COV.2.S vaccine could not be assessed due to a small number of exposures. We recommend COVID-19 vaccination regardless of pregnancy status.

3. Joint statement from ASA/APSF on preoperative COVID-19 testing regardless of vaccination status (August 2021)

Given the ongoing and evolving pandemic, an updated joint statement from the American Society of Anesthesiologists and the Anesthesia Patient Safety Foundation recommends that in areas of high COVID-19 prevalence, all patients undergoing a nonemergency procedure with the potential to generate aerosols should continue to have preoperative testing for SARS-CoV-2 irrespective of vaccination status, using a nucleic acid amplification test (e.g., 
RT-PCR) and ideally $\leq 3$ days before the procedure [4]. Elective surgical procedures should be delayed in patients who test positive. In addition, all patients who screen positive for symptoms of COVID-19 preoperatively should be referred for evaluation.

4. Timing of delivery in fetal growth restriction (August 2021)

There is little consensus about the optimum time to deliver the fetus with growth restriction (FGR). In a retrospective cohort study, infants who were severely small for gestational age (SGA, birth weight $<3$ rd percentile) and delivered early for suspected FGR had poorer school outcomes in grades 3, 5 and 7 compared with infants with the same degree of SGA who were not suspected of having FGR (mean gestational age at birth: 37.9 versus 39.4 weeks) [5]. Although these findings suggest that avoiding early delivery of suspected FGR when safe to do so may improve school performance of SGA infants, other differences between the two groups may account for the better outcome.

5. Fertility and pregnancy outcomes among female surgeons (July 2021)

In a study that surveyed 692 female surgeons and 158 male surgeons and their female nonsurgeon partners regarding fertility and pregnancy history, female surgeons were more than twice as likely to report a pregnancy loss compared with the general population and nearly twice as likely to report pregnancy complications compared with the female nonsurgeon partners, in adjusted analysis [6]. Compared with male surgeons, female surgeons were more likely to delay childbearing because of training, use assisted reproductive technology and have fewer children. As more females enter the surgical workforce, these data can inform development of policy that supports pregnancy and family building goals for all surgeons.

6. Delayed cord clamping versus cord milking in preterm infants (April 2021)

Delayed cord clamping (DCC) facilitates the physiologic transition from fetal to newborn life, particularly in preterm infants. Cord milking (CM) is an alternative, but a previous meta-analysis of randomized trials found that it increased the risk for severe (grade 3 or 4) intraventricular hemorrhage (IVH). However, in a new network meta-analysis, DCC and CM resulted in similar odds of severe IVH and other neonatal outcomes [7]. Interpretation of available data is limited by differences in how CM was performed and the gestational age range for preterm birth among trials. Although, when feasible, we prefer DCC to $\mathrm{CM}$, these data suggest equipoise between
DCC and CM in preterm births. CM should not interfere with delivery when immediate pediatric assistance is needed (e.g., neonatal depression or thick meconium) or if cord blood collection is planned.

\section{References}

1. Badr DA, Picone O, Bevilacqua E, et al. Severe acute respiratory syndrome coronavirus 2 and pregnancy outcomes according to gestational age at time of infection. Emerg Infect Dis. 2021;27:2535.

2. Zauche LH, Wallace B, Smoots AN, et al. Receipt of mRNA Covid-19 vaccines and risk of spontaneous abortion. N Engl J Med. 2021;385(16):1533-5. https://doi.org/10.1056/NEJMc21138 91. (Epub 2021 Sep 8)

3. Kharbanda EO, Haapala J, DeSilva M, et al. Spontaneous abortion following COVID-19 vaccination during pregnancy. JAMA. 2021;326(16):1629-31. https://doi.org/10.1001/jama.2021.15494.

4. https://www. asahq.org/about-asa/newsroom/news-relea ses/2021/08/asa-and-apsf-statement-on-perioperative-testi ng-for-the-covid-19-virus.

5. Selvaratnam RJ, Wallace EM, Wolfe R, et al. Association between iatrogenic delivery for suspected fetal growth restriction and childhood school outcomes. JAMA. 2021;326:145.

6. Rangel EL, Castillo-Angeles M, Easter SR, et al. Incidence of infertility and pregnancy complications in US female surgeons. JAMA Surg. 2021;156(10):905-915. https://doi.org/10.1001/jamasurg. 2021.3301 .

7. Jasani B, Torgalkar R, Ye XY, et al. Association of umbilical cord management strategies with outcomes of preterm infants: a systematic review and network meta-analysis. JAMA Pediatr. 2021;175:e210102.

Publisher's Note Springer Nature remains neutral with regard to jurisdictional claims in published maps and institutional affiliations.

\section{About the Author}

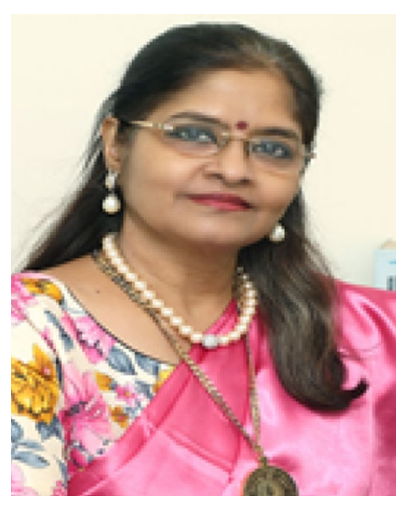

Dr. Geetha Balsarkar is a Professor at Seth G.S. Medical College and Nowrosjee Wadia Maternity Hospital, Mumbai, India. She is a Librarian of Mumbai Obstetrics Gynecology Society, President of Menopause Society, Mumbai Branch, President of AMWI, Mumbai Branch, and Editor-InChief, Journal of Obstetrics and Gynecology of India, FOGSI Journal. She has undergone training for the PPIUCD program and is also the faculty member of many training programmes as well. She has over 18 years of teaching experience and is actively working on FIGO FOGSI Project of PPIUCD in India. She is a recipient of the prestigious Hargobind Foundation for study of Fetal Medicine in 2008 at St. George's Hospital, London. 